\title{
Measuring the masses of a pair of semi-invisibly decaying particles in central exclusive production with forward proton tagging
}

\author{
L. A. Harland-Lang ${ }^{1}$, C. H. Kom ${ }^{2}$, K. Sakurai ${ }^{3,4}$, and W. J. Stirling ${ }^{1}$ \\ 1 Cavendish Laboratory, University of Cambridge, CB3 0HE, UK \\ 2 Department of Mathematical Sciences, University of Liverpool, Liverpool L69 3BX, UK \\ 3 Deutsches Elektronen-Synchrotron DESY, 22603 Hamburg, Germany \\ 4 Department of Physics, Nagoya University, Nagoya 464-8602, Japan
}

February 7, 2012

\begin{abstract}
We discuss how the mass of new physics particles involved in a pair of short decay chains leading to two invisible particles, for example slepton pair production, followed by the decay into two leptons and two neutralinos, may be measured in central exclusive production (CEP) with forward proton tagging. We show how the existing mass measurement strategies in CEP may be improved by making full use of the mass-shell constraints, and demonstrate that, with around 30 signal events, the masses of the slepton and neutralino can be measured with an accuracy of a few $\mathrm{GeV}$.
\end{abstract}

\section{Introduction}

Many theories beyond the Standard Model (SM) include a dark matter (DM) candidate as part of the mass spectrum. Typically, the stability of the DM candidate is the result of an assumed global $\mathcal{Z}_{2}$ parity, under which the new (SM) particles are odd (even). For example, in the minimal supersymmetric extension of the SM (MSSM), this is the so-called $\mathrm{R}$-parity $\mathcal{R}_{p}$, while in models with large extra dimensions, this could be a Kaluza-Klein (KK) parity.

If such a DM candidate is produced at the Large Hadron Collider (LHC), its presence could be revealed through the observation of large missing transverse energy. The $\mathcal{Z}_{2}$ parity implies that the $\mathrm{DM}$ candidate will be pairproduced at the LHC. This leads to complications in measuring its properties, in particular its mass, since only the vector sum of the transverse momenta of the two DM candidates in an event can be measured.

Strategies to circumvent this problem abound in the literature [1 26] 1] Many of these assume the existence of certain long decay chains. An important example in the MSSM is the pair production of squarks $(\tilde{q})$, followed by the decay chain

$$
\begin{aligned}
& \tilde{q} \rightarrow q \tilde{\chi}_{2}^{0}, \\
& \tilde{\chi}_{2}^{0} \rightarrow l^{ \pm} \tilde{l}^{\mp}, \\
& \\
& \quad \tilde{l}^{\mp} \rightarrow l^{\mp} \tilde{\chi}_{1}^{0},
\end{aligned}
$$

where $\tilde{\chi}_{1,2}^{0}$ are the neutralinos ( $\tilde{\chi}_{1}^{0}$ is the DM candidate), and $\tilde{l}$ is a slepton. The existence of more constraints,

\footnotetext{
1 See also Ref. 27] for a review.
}

e.g. mass-shell conditions and dilepton invariant mass edge measurements, than undetermined 4-momenta 2 would allow simultaneous determination of the mass of the new particles. These long decay chains imply various challenges for the relevant mass measurement techniques, e.g. correct identification of the decay topology and combinatoric problems in assigning the identified final state particles to appropriate stages of the decay chains, and so it is advantageous to consider alternative methods to complement existing strategies.

The central exclusive production (CEP) of a system $X$ can be written in the form

$$
p p \rightarrow p+X+p,
$$

where the ' + ' signs represent the presence of large rapidity gaps. In this type of reaction, only the system $X$ is produced in the central detector, with no additional hadronic activity in the absence of pile-up, see for example Ref. [28, 29] (and references therein) for a review. The outgoing protons scatter at small angles and remain intact, so that additional kinematic information can be obtained by installing proton tagging detectors far from the interaction point [30]. In particular, in the presence of these detectors the momenta of the outgoing protons can be measured precisely, and therefore the longitudinal momentum and invariant mass of the central system $\mathrm{X}$ can be accurately determined.

\footnotetext{
${ }^{2}$ In the present context, there are $6(=8-2)$ unknowns: the four momenta of the two $\tilde{\chi}_{1}^{0}(8)$, minus the measured missing transverse momenta (2).
} 
In this paper, we present a mass measurement method utilising the additional longitudinal kinematic information in the exclusive two-photon production of a pair of charged particles $\left(X_{1}^{+}, X_{2}^{-}\right)$,

$$
\begin{aligned}
p p \rightarrow p+ & \gamma \gamma+p, \\
& \gamma \gamma \rightarrow X_{1}^{+} X_{2}^{-},
\end{aligned}
$$

followed by the decay

$$
X^{ \pm} \rightarrow x^{ \pm}+\chi
$$

where $x$ is a visible particle (or more generally, fully reconstructed system of particles), and $\chi$ is an invisible particle that is not seen in the detector. For concreteness, we will consider the specific case of the exclusive two-photon production of a pair of sleptons $\left(\tilde{l}_{1}^{+}, \tilde{l}_{2}^{-}\right)$, followed by the decay into leptons $\left(l_{1}^{+}, l_{2}^{-}\right)$and the lightest neutralinos $\left(\tilde{\chi}_{1}, \tilde{\chi}_{2}\right)$ (note the different notations compared with Eq. (11)), i.e.

$$
\begin{aligned}
p p \rightarrow p+\gamma \gamma+ & p \\
\gamma \gamma \rightarrow & \tilde{l}_{1}^{+} \tilde{l}_{2}^{-}, \\
& \tilde{l}_{1}^{+} \tilde{l}_{2}^{-} \rightarrow l_{1}^{+} l_{2}^{-} \tilde{\chi}_{1} \tilde{\chi}_{2},
\end{aligned}
$$

leading to a signature with two tagged protons, an oppositesign same-flavour lepton pair and missing 4-momentum with no additional jet activity. The irreducible SM background comes from the production of $W$ pairs, i.e.

$$
\begin{aligned}
p p \rightarrow p+\gamma \gamma+ & p \\
\gamma \gamma \rightarrow & W_{1}^{+} W_{2}^{-}, \\
& W_{1}^{+} W_{2}^{-} \rightarrow l_{1}^{+} l_{2}^{-} \nu_{1} \bar{\nu}_{2} .
\end{aligned}
$$

Compared to the long decay chain discussed in Eq. (1), the two-photon production process benefits from a clean, purely leptonic signature, with no combinatoric ambiguity. Furthermore, assuming the slepton only decays into the lightest neutralino, the process is essentially a two scale problem that depends on the (true) masses of the slepton and the lightest neutralino, $\left(m_{\tilde{l}}^{\text {true }}, m_{\tilde{\chi}}^{\text {true }}\right)$, together with discrete parameters such as the particles' charges and spins.

The irreducible SM background is also under good experimental control. This is because in the case of the signal, two-photon production must create two same-flavour sleptons. In the absence of large lepton flavour violation, this will lead to two same-flavour leptons in the final state. For the SM background there is no such constraint on the flavour of the final-state leptons: a measurement of the opposite flavour final states $e^{ \pm} \mu^{\mp}$ would therefore allow a cross check of the background determination.

However, the cross section for the exclusive two-photon production of slepton pairs is small when compared to the inclusive production cross section from hard inelastic scattering, which is roughly two orders of magnitude larger [31, 32]: as a result, mass measurement in this central exclusive channel is only realistic at the $14 \mathrm{TeV}$ LHC for $m_{\tilde{l}}$ up to about $200 \mathrm{GeV}$. However if the coloured sparticles, i.e. the squarks and the gluinos, are too heavy to be produced copiously at the LHC, the presence and properties of light colour singlet sparticles may be best measured via two-photon production. If (as we hope) the coloured sparticles are produced in abundance, and long decay chains are observed, the present method might still be used as an independent check or to provide external constraints on the long decay chains.

The system in Eq. (5) has four unknowns: only the vector sum of the 4 -momenta of $\tilde{\chi}_{1}$ and $\tilde{\chi}_{2}$ is measurable. Given a consistent mass hypothesi ${ }^{3}\left(m_{\tilde{l}}, m_{\tilde{\chi}}\right)$, the four quadratic mass shell constraints allow the system of equations to be solved analytically, up to a two-fold degeneracy. As a result, a $\left(m_{\tilde{l}}, m_{\tilde{\chi}}\right)$ mass region consistent with the measured 4-momenta can be obtained on an event-by-event basis. We believe this consistent mass regions contains all information that can be derived from the measured 4-momenta. Neglecting finite detector resolution effects, the (different) consistent mass region from each event must contain the true mass point $\left(m_{\tilde{l}}^{\text {true }}, m_{\tilde{\chi}}^{\text {true }}\right)$. Combining the consistent regions from a number of events will develop a peaking structure around this point, hence in principle allowing determination of the true masses.

In determining the true masses and in separating the signal from SM background, we find the maximum values of $m_{\tilde{l}}\left(m_{\tilde{l}}^{\max }\right)$ and $m_{\tilde{\chi}}\left(m_{\tilde{\chi}}^{\max }\right)$ within the consistent mass region particularly useful. This is because, by construction, $m_{\tilde{l}}^{\max }$ and $m_{\tilde{\chi}}^{\max }$ are bounded from below by $m_{\tilde{l}}^{\text {true }}$ and $m_{\tilde{\chi}}^{\text {true }}$ respectively. They are also bounded from above by half the $\gamma \gamma$ invariant mass $\left(m_{\gamma \gamma}\right)$ and half the missing energy $\left(E_{\text {miss }}\right)$. An additional advantage is the relative ease in constructing likelihood functions from $m_{\tilde{l}}^{\max }$ and $m_{\tilde{\chi}}^{\max }$, which could be particularly useful given a small data sample, as is likely the case in light of the small twophoton production cross section.

The main aim of this paper is to study how well the slepton and neutralino masses may be determined using $m_{\tilde{l}}^{\max }$ and $m_{\tilde{\chi}}^{\max }$ computed in each event. These masses can also be estimated from $m_{\gamma \gamma}$ and $E_{\text {miss }}$ [33, 34], which have threshold values at $2 m_{\tilde{l}}^{\text {true }}$ and $2 m_{\tilde{\chi}}^{\text {true }}$ respectively. We shall compare the two methods and illustrate the improvements that can be made by making use of the massshell constraints. The potential for measuring properties of the SM Higgs boson 35, 36], long-lived gluinos 37], stops 38], MSSM Higgs 38, 39], NMSSM Higgs 40] and other BSM Higgs scenarios [41, 42] in the central exclusive mode with tagged forward protons have also been discussed in the literature.

Note that the decay chain considered can be applied to new physics models with different particle spins, for example the pair production of charged spin $-1 / 2$ fermions followed by the decay into neutral spin-1 bosons, as might be expected from a KK model. In fact, it can be shown that, given the same masses and charges, the production cross section for spin-0 bosons is the smallest when compared with spin-1/2 fermions and spin-1 bosons, see Fig. 1 In this paper, we shall concentrate on the first case, which is

\footnotetext{
3 The technical definition of a consistent mass hypothesis
} will be given in the next section. 


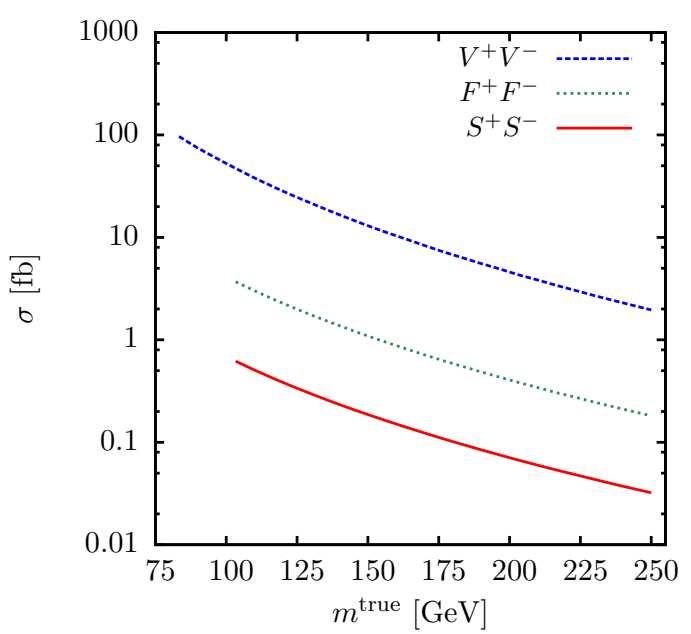

Fig. 1. Cross sections for pair production of spin-1 $\left(V^{+} V^{-}\right)$, spin-1/2 $\left(F^{+} F^{-}\right)$and spin-0 $\left(S^{+} S^{-}\right)$charged particles in central exclusive two-photon production as a function of the particle mass $\left(m^{\text {true }}\right)$ at the $14 \mathrm{TeV}$ LHC, calculated using the PhoCEP Monte Carlo event generator.

the most conservative, and use the SUSY decay chain in Eq. (5) as our working example.

The paper is organised as follows. In Section 2, we present the analytic solutions consistent with the 4-momenta measurable in Eq. (5), and derive the quantities $m_{\tilde{l}}^{\max }$ and $m_{\tilde{\chi}}^{\max }$. We then present the numerical results in Section 3 . and compare between the performance of $\left(m_{\tilde{l}}^{\max }, m_{\tilde{\chi}}^{\max }\right)$ and $\left(m_{\gamma \gamma}, E_{\text {miss }}\right)$ in determining $\left(m_{\tilde{l}}^{\text {true }}, m_{\tilde{\chi}}^{\text {true }}\right)$, after including the CEP dynamics simulated by a new event generator PhoCEP [43]. This is followed by an estimate of the quantities $\left(m_{\tilde{l}}^{\text {true }}, m_{\tilde{\chi}}^{\text {true }}\right)$ using the likelihood method advertised above assuming an integrated luminosity of $300 \mathrm{fb}^{-1}$ at the $14 \mathrm{TeV}$ LHC, which is the main result of our paper. We conclude in Section 4.

\section{The consistent solutions}

The 4-momenta of the particles in the decay chain in Eq. (5) are subjected to the constraints

$$
\begin{aligned}
p_{\gamma \gamma} & =p_{\tilde{l}_{1}}+p_{\tilde{l}_{2}}, \\
p_{\tilde{l}_{i}} & =p_{\tilde{\chi}_{i}}+p_{l_{i}}, \\
p_{\tilde{l}_{i}}^{2} & =m_{\tilde{l}}^{2}, \\
p_{\tilde{\chi}_{i}}^{2} & =m_{\tilde{\chi}}^{2},
\end{aligned}
$$

where $(i=1,2)$ and $p_{\gamma \gamma}$ is the 4 -momentum vector sum of the two $\gamma$ 's. For simplicity, the sign of the sleptons and leptons are dropped. In the above expressions, only $p_{\gamma \gamma}$ and $p_{l_{i}}$ are directly measurable. The 4 -momenta of the SUSY particles, $p_{\tilde{l}_{i}}$ and $p_{\tilde{\chi}_{i}}$, can be expressed as linear combinations of $p_{\gamma \gamma}, p_{l_{i}}$ and $P$, a space-like 4-momentum vector defined by

$$
P_{\mu} \equiv \epsilon_{\mu \nu \rho \sigma} p_{\gamma \gamma}^{\nu} p_{l_{1}}^{\rho} p_{l_{2}}^{\sigma}
$$

which has the property

$$
P \cdot p_{\gamma \gamma}=P \cdot p_{l_{1}}=P \cdot p_{l_{2}}=0 \text {. }
$$

Eq. (7) implies

$$
\begin{aligned}
p_{\tilde{\chi}_{1}} & =\frac{1}{2}(1-a) p_{\gamma \gamma}-\frac{1}{2}(b+1) p_{l_{1}}+\frac{1}{2}(c-1) p_{l_{2}}-d P, \\
p_{\tilde{\chi}_{2}} & =\frac{1}{2}(1+a) p_{\gamma \gamma}+\frac{1}{2}(b-1) p_{l_{1}}-\frac{1}{2}(c+1) p_{l_{2}}+d P, \\
p_{\tilde{l}_{1}} & =\frac{1}{2}(1-a) p_{\gamma \gamma}-\frac{1}{2}(b-1) p_{l_{1}}+\frac{1}{2}(c-1) p_{l_{2}}-d P \\
p_{\tilde{l}_{2}} & =\frac{1}{2}(1+a) p_{\gamma \gamma}+\frac{1}{2}(b-1) p_{l_{1}}-\frac{1}{2}(c-1) p_{l_{2}}+d P
\end{aligned}
$$

with mass--shell conditions

$$
\begin{aligned}
m_{\tilde{\chi}_{1}}^{2}= & \frac{1}{4}(1-a)^{2} m_{\gamma \gamma}^{2}+d^{2} P^{2}-\frac{1}{2}(1-a)(b+1) p_{\gamma \gamma} \cdot p_{l_{1}} \\
& +\frac{1}{2}(1-a)(c-1) p_{\gamma \gamma} \cdot p_{l_{2}}-\frac{1}{2}(b+1)(c-1) p_{l_{1}} \cdot p_{l_{2}}, \\
m_{\tilde{\chi}_{2}}^{2}= & \frac{1}{4}(1+a)^{2} m_{\gamma \gamma}^{2}+d^{2} P^{2}+\frac{1}{2}(1+a)(b-1) p_{\gamma \gamma} \cdot p_{l_{1}} \\
& -\frac{1}{2}(1+a)(c+1) p_{\gamma \gamma} \cdot p_{l_{2}}-\frac{1}{2}(b-1)(c+1) p_{l_{1}} \cdot p_{l_{2}}, \\
m_{\tilde{l}_{1}}^{2}= & \frac{1}{4}(1-a)^{2} m_{\gamma \gamma}^{2}+d^{2} P^{2}-\frac{1}{2}(1-a)(b-1) p_{\gamma \gamma} \cdot p_{l_{1}} \\
& +\frac{1}{2}(1-a)(c-1) p_{\gamma \gamma} \cdot p_{l_{2}}-\frac{1}{2}(b-1)(c-1) p_{l_{1}} \cdot p_{l_{2}}, \\
m_{\tilde{l}_{2}}^{2}= & \frac{1}{4}(1+a)^{2} m_{\gamma \gamma}^{2}+d^{2} P^{2}+\frac{1}{2}(1+a)(b-1) p_{\gamma \gamma} \cdot p_{l_{1}} \\
& -\frac{1}{2}(1+a)(c-1) p_{\gamma \gamma} \cdot p_{l_{2}}-\frac{1}{2}(b-1)(c-1) p_{l_{1}} \cdot p_{l_{2}},
\end{aligned}
$$

where the parameters $a, b, c$ and $d$ are to be determined. For simplicity, we have neglected the lepton mass $m_{l}$ throughout. The full solution including general mass values can be found in Ref. 44].

Here $m_{\tilde{\chi}}$ and $m_{\tilde{l}}$ need not be the true masses, but input parameters consistent with a positive $d^{2}$. The structure of the system is such that upon subtracting the different expressions in Eq. (11), the additive $d^{2} P^{2}$ term and the terms bilinear or quadratic in $(a, b, c)$ drop out. This leads to three equations linear in $(a, b, c)$. Defining the quantities

$$
\begin{array}{ll}
\Lambda_{\Delta} \equiv \frac{\Delta m_{\tilde{l} \tilde{\chi}}^{2}}{p_{l_{1}} \cdot p_{l_{2}}}, & \Lambda_{1} \equiv \frac{p_{\gamma \gamma} \cdot p_{l_{1}}}{p_{l_{1}} \cdot p_{l_{2}}} \\
\Lambda_{2} \equiv \frac{p_{\gamma \gamma} \cdot p_{l_{2}}}{p_{l_{1}} \cdot p_{l_{2}}}, & \Lambda_{\gamma \gamma} \equiv \frac{m_{\gamma \gamma}^{2}}{p_{l_{1}} \cdot p_{l_{2}}}
\end{array}
$$

where

$$
\Delta m_{\tilde{l} \tilde{\chi}}^{2} \equiv m_{\tilde{l}}^{2}-m_{\tilde{\chi}}^{2},
$$


the solutions for the parameters $(a, b, c)$ are given by

$$
\begin{aligned}
& a=\Lambda_{\Delta} \frac{\left(\Lambda_{2}-\Lambda_{1}\right)}{\Lambda_{\gamma \gamma}-2 \Lambda_{1} \Lambda_{2}}, \\
& b=1-\Lambda_{2}+\Lambda_{\Delta} \frac{\Lambda_{\gamma \gamma}-\Lambda_{2}\left(\Lambda_{1}+\Lambda_{2}\right)}{\Lambda_{\gamma \gamma}-2 \Lambda_{1} \Lambda_{2}}, \\
& c=1-\Lambda_{1}+\Lambda_{\Delta} \frac{\Lambda_{\gamma \gamma}-\Lambda_{1}\left(\Lambda_{1}+\Lambda_{2}\right)}{\Lambda_{\gamma \gamma}-2 \Lambda_{1} \Lambda_{2}} .
\end{aligned}
$$

Inserting Eq. (14) into Eq. (11), all four mass constraints give

$$
\Lambda_{\tilde{\chi}}=c_{a} \Lambda_{\Delta}^{2}+c_{b} \Lambda_{\Delta}+c_{c}+d^{2} \Lambda_{P}
$$

where

$$
\Lambda_{\tilde{\chi}} \equiv \frac{m_{\tilde{\chi}}^{2}}{p_{l_{1}} \cdot p_{l_{2}}}, \quad \Lambda_{\tilde{l}} \equiv \frac{m_{\tilde{l}}^{2}}{p_{l_{1}} \cdot p_{l_{2}}}, \quad \Lambda_{P} \equiv \frac{P^{2}}{p_{l_{1}} \cdot p_{l_{2}}},
$$

and

$$
\begin{aligned}
c_{a} & =\frac{1}{4} \frac{\left(\Lambda_{1}+\Lambda_{2}\right)^{2}-2 \Lambda_{\gamma \gamma}}{\Lambda_{\gamma \gamma}-2 \Lambda_{1} \Lambda_{2}}, \\
c_{b} & =\frac{1}{2}\left(\Lambda_{1}+\Lambda_{2}-2\right), \\
c_{c} & =\frac{1}{4}\left(\Lambda_{\gamma \gamma}-2 \Lambda_{1} \Lambda_{2}\right) .
\end{aligned}
$$

Note that in Eq. (15), $c_{a}, c_{b}, c_{c}$ and $\Lambda_{P}$ are constructed from directly measureable $4-$ momenta. A mass hypothesis $\left(m_{\tilde{l}}, m_{\tilde{\chi}}\right)$ is consistent with the measured $4-$ momenta if it results in a positive $d^{2}$, in which case a two-fold degenerate solution for $\left(p_{\tilde{\chi}_{1}}, p_{\tilde{\chi}_{2}}, p_{\tilde{l}_{1}}, p_{\tilde{l}_{2}}\right)$ corresponding to $\pm|d|$ is obtained.

The boundary of consistent solutions on the $\left(m_{\tilde{l}}, m_{\tilde{\chi}}\right)$ plane is given by Eq. (15) by setting $d=0$. To see this, note that since $P$ is space-like, $\Lambda_{P}<0$. This means that for a given $\Lambda_{\Delta}, \Lambda_{\tilde{\chi}}$ decreases as $d^{2}$ increases. An upper boundary on the $\left(\Delta m_{\tilde{l} \tilde{\chi}}^{2}, m_{\tilde{\chi}}^{2}\right)$ plane therefore has $d^{2}=$ 0 . The consistent region is bounded from above by this quadratic curve, and below by the $x$-axis, i.e. $m_{\tilde{\chi}}^{2}=0$, which can be transformed into a boundary in the $\left(m_{\tilde{l}}, m_{\tilde{\chi}}\right)$ plane. Within the boundary, $d^{2}>0$. By construction, the solutions are time-like.

More observations on the properties of the consistent solutions can be made. First, the sign of the energy component of the two solutions (for $\pm|d|$ ) must be the same, since it is always possible to boost to a frame where the energy component of the space-like vector $P$ is zero, in which case the two solutions have the same energies. Second, since the consistent solutions are continuous functions of $m_{\tilde{\chi}}$ and $m_{\tilde{l}}$, it follows that the energies of all consistent solutions must have the same sign. The energies must then be positive, due to the fact that the true masses must be a consistent solution, and hence lies within the boundary. Third, the solution is clearly symmetric when exchanging $\Lambda_{1}$ and $\Lambda_{2}$. This means that the boundary obtained for the mass-shell conditions for $p_{\tilde{\chi}_{1}}$ and $p_{\tilde{\chi}_{2}}$ must be the same, consistent with the fact that both constraints lead to Eq. (15).
As discussed in Section 1, the consistent mass region may be characterised by the maximum values of $m_{\tilde{\chi}}$ and $m_{\tilde{l}}$, i.e. $m_{\tilde{\chi}}^{\max }$ and $m_{\tilde{l}}^{\max }$. They correspond to the stationary values of $\Lambda_{\tilde{\chi}}$ and $\Lambda_{\tilde{l}}$ as functions of $\Lambda_{\Delta}$ in Eq. (15). The results are

$$
\begin{aligned}
& \left(m_{\tilde{\chi}}^{\max }\right)^{2}=\left(p_{l_{1}} \cdot p_{l_{2}}\right) \times\left(c_{c}-\frac{c_{b}^{2}}{4 c_{a}}\right), \\
& \left(m_{\tilde{l}}^{\max }\right)^{2}=\left(p_{l_{1}} \cdot p_{l_{2}}\right) \times\left(c_{c}-\frac{\left(c_{b}+1\right)^{2}}{4 c_{a}}\right) .
\end{aligned}
$$

By construction, $m_{\tilde{\chi}}^{\max }$ and $m_{\tilde{l}}^{\max }$ are bounded from below by the true $m_{\tilde{\chi}}$ and $m_{\tilde{l}}$ respectively.

So far we have shown that analytic boundaries containing mass hypotheses consistent with the measured 4 momenta can be obtained on an event-by-event basis. In the next section, we present examples showing the densities of consistent solutions given a large number of events, and show the extent to which the quantities $m_{\tilde{l}}^{\max }$ and $m_{\tilde{\chi}}^{\max }$ can be used to determine the true masses, crucially in a small event sample.

\section{Numerical study}

Our numerical study is performed using the new Monte Carlo (MC) event generator PhoCEP [43]. It simulates the two-photon exclusive production of a $W^{+} W^{-}$pair and a charged scalar pair, $S^{+} S^{-}$, via the decay chains given in Eqs. (5) and (6), as well as the production of two charged fermions, $F^{+} F^{-}$, although we do not discuss this process in detail in this paper. The equivalent photon approximation [30, 45] is used to calculate the photon luminosity $\mathrm{d} L_{\gamma \gamma} / \mathrm{d} W$ due to photon emission from the incoming protons, and this is then combined with the known $\gamma \gamma \rightarrow X$ $\left(X=W^{+} W^{-}, S^{+} S^{-}, F^{+} F^{-}\right)$subprocess cross sections to give the full CEP cross section

$$
\frac{\mathrm{d} \sigma_{p p \rightarrow p X p}}{\mathrm{~d} \Omega}=\int \frac{\mathrm{d} \sigma_{\gamma \gamma \rightarrow X}(W)}{\mathrm{d} \Omega} \frac{\mathrm{d} L^{\gamma \gamma}}{\mathrm{d} W} \mathrm{dW},
$$

where $W$ is the $\gamma \gamma$ cms energy. The produced particles are then decayed according to the decay chains given in Eqs. (5) and (6), and in the case of $W^{+} W^{-}$production full spin correlations for the subsequent decay are included. The MC generates unweighted events, and full kinematic cuts on the final state particles and outgoing protons can readily be implemented. The survival factor $S^{2}$, representing the probability that the outgoing protons do not undergo soft rescattering and therefore spoil the exclusivity of the final state, is estimated to be roughly $90 \%$ for twophoton exclusive production [29], and for simplicity is not included in the MC. The differential cross sections for the $\gamma \gamma \rightarrow X$ subprocesses are summarised in Appendix 4 .

In the following, we present numerical results of the methods discussed in Section 2. For concreteness, we focus on a SUSY model

$$
\text { - SUSY model: }\left(m_{\tilde{l}}^{\text {true }}, m_{\tilde{\chi}}^{\text {true }}\right)=(150,100) \mathrm{GeV}
$$




\begin{tabular}{|c|c|c|}
\hline & Before cuts (fb) & After cuts $(\mathrm{fb})$ \\
\hline SM & 1.00 & 0.72 \\
SUSY & 0.15 & 0.13 \\
\hline
\end{tabular}

Table 1. Cross sections for the SUSY model with $\left(m_{\tilde{l}}^{\text {true }}, m_{\tilde{\chi}}^{\text {true }}\right)=(150,100) \mathrm{GeV}$ and the SM background at the $14 \mathrm{TeV}$ LHC before and after the lepton cuts $-2.5<\eta_{l}<2.5$ and $p_{T}>10 \mathrm{GeV}$. Cuts on the fractional momentum loss of the outgoing protons $0.0015<\xi_{i}<0.15$, are applied in both cases. Branching fraction of the $W$ into one lepton flavour is included.

and compare with results for SM $W^{+} W^{-}$pair production, which constitutes an irreducible background to the SUSY process. For the SUSY model, the slepton is assumed to always decay into a lepton and a neutralino. The branching fraction of the SM $W$ into one lepton flavour is taken as $10.8 \%$ [46].

In our simulation, a final state proton is assumed to be tagged with $100 \%$ efficiency if the fractional momentum loss lies within the range

$-0.0015<\xi_{i}<0.15 i=1,2$,

which corresponds to the expected coverage under the assumption that roman pot detectors are installed at a distance of 220 and $420 \mathrm{~m}$ from interaction point, see for example Ref. [47].

The following lepton pseudo-rapidity $\left(\eta_{l}\right)$ and transverse momentum $\left(p_{T}\right)$ cuts

$$
--2.5<\eta_{l}<2.5
$$$$
-p_{T}>10 \mathrm{GeV}
$$

are applied to the opposite-sign same-flavour lepton pairs. We assume $100 \%$ detection efficiency if a lepton passes the above cuts. The cross sections before and after the lepton cuts at the $14 \mathrm{TeV}$ LHC are displayed in Table 1.

In Fig. 2, the density of the consistent $\left(m_{\tilde{l}}, m_{\tilde{\chi}}\right)$ regions are shown. In both the SUSY and SM samples, a peaking structure located in the vicinity of the true mass point develops. Finite detector resolution effects will smear the spike structure slightly. However, given the LHC's ability to measure accurately the 4-momenta of the forward protons (in the presence of proton taggers) and the charged leptons, the smearing effects should be relatively mild. The position of the high density regions are significantly different such that the SUSY signal can be easily separated from the background.

Next we show in Fig. 3 scatter plots for $m_{\tilde{\chi}}^{\max }$ and $m_{\tilde{l}}^{\max }$, each of which contains 10,000 events. Recall that, by construction these two quantities are bounded from below by $m_{\tilde{\chi}}^{\text {true }}$ and $m_{\tilde{l}}^{\text {true }}$. As we can see, in both the SUSY and SM samples $\left(m_{\tilde{l}}^{\max }, m_{\tilde{\chi}}^{\max }\right)$ cluster close to the true mass point $\left(m_{\tilde{l}}^{\text {true }}, m_{\tilde{\chi}}^{\text {true }}\right)$. This indicates that a good estimation of the true mass parameters and identification of the presence of a SUSY signal from the SM background could in principle be achievable.

To show the enhancement obtained by utilising the additional kinematic information, in particular in mass determination, we compare our results with an alternative

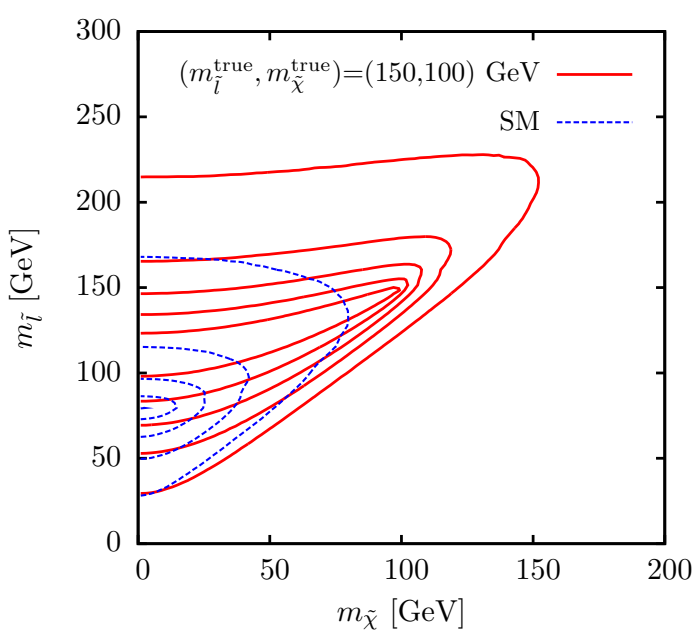

Fig. 2. Density of the consistent mass regions for a SUSY (solid red) and SM (dashed blue) sample. From the outermost to the innermost, each set of contours delineate parameter regions with probability density larger than $0.1,0.3,0.5$, 0.7 and 0.9. The mass parameters for the SUSY model are $\left(m_{\tilde{l}}^{\text {true }}, m_{\tilde{\chi}}^{\text {true }}\right)=(150,100) \mathrm{GeV}$. No detector smearing is included.

method which measures the end points of the missing energy $\left(E_{\text {miss }}\right)$ and $m_{\gamma \gamma}$ distributions [33]. The end point values of these distributions are at $2 m_{\tilde{\chi}}^{\text {true }}$ and $2 m_{\tilde{l}}^{\text {true }}$ respectively. These distributions take into account the sum of longitudinal momenta and energy of the neutralinos that can be measured in CEP with tagged forward protons, but do not include information from mass constraints.

In Fig. 4. we show the $m_{\tilde{\chi}}^{\max }$ and $m_{\tilde{l}}^{\max }$ distributions. The $E_{\text {miss }}$ and $m_{\gamma \gamma}$ distributions are displayed in Fig. 5. Compared to the $m_{\tilde{\chi}}^{\max }$ and $m_{\tilde{l}}^{\max }$ distributions, the $E_{\text {miss }}$ and $m_{\gamma \gamma}$ distributions are generally broader. This qualitative difference is due to the fact that both $E_{\text {miss }} / 2$ and $m_{\tilde{\chi}}^{\max }\left(m_{\gamma \gamma} / 2\right.$ and $\left.m_{\tilde{l}}^{\max }\right)$ are bounded from below by $m_{\tilde{\chi}}^{\text {true }}\left(m_{\tilde{l}}^{\text {true }}\right)$, while $m_{\tilde{\chi}}^{\max }\left(m_{\tilde{l}}^{\max }\right)$ is in addition bounded from above by $E_{\text {miss }} / 2\left(m_{\gamma \gamma} / 2\right)$. To compare the difference between these two methods over a range of masses, we show two additional SUSY models, specified by $\left(m_{\tilde{l}}^{\text {true }}, m_{\tilde{\chi}}^{\text {true }}\right)=$ $(100,50) \mathrm{GeV}$ and $\left(m_{\tilde{l}}^{\text {true }}, m_{\tilde{\chi}}^{\text {true }}\right)=(200,100) \mathrm{GeV}$ in addition to the 'reference' SUSY model and the SM background. We see that, compared with the $\left(E_{\text {miss }}, m_{\gamma \gamma}\right)$ distributions, the $\left(m_{\tilde{\chi}}^{\max }, m_{\tilde{l}}^{\max }\right)$ distributions benefit from a better signal-to-background ratio (S/B) in the signal region, which improves as the mass scale increases. Here, the signal region for a distribution is defined as the region above the lower threshold value determined by $\left(m_{\tilde{l}}^{\text {true }}, m_{\tilde{\chi}}^{\text {true }}\right)$. For example, for $\left(m_{\tilde{l}}^{\text {true }}, m_{\tilde{\chi}}^{\text {true }}\right)=(150,100) \mathrm{GeV}$, the S/B ratio in the signal region is approximately 1 for both $m_{\tilde{l}}^{\max }$ and $m_{\tilde{\chi}}^{\max }$ distributions, while it is approximately $1 / 4$ and $1 / 3$ for the $E_{\text {miss }}$ and $m_{\gamma \gamma}$ distributions respectively. Importantly, the distribution of the signal $E_{\text {miss }}$ distributions near the endpoint becomes increasingly smooth as the mass scale increases, leading to possibly large uncertain- 

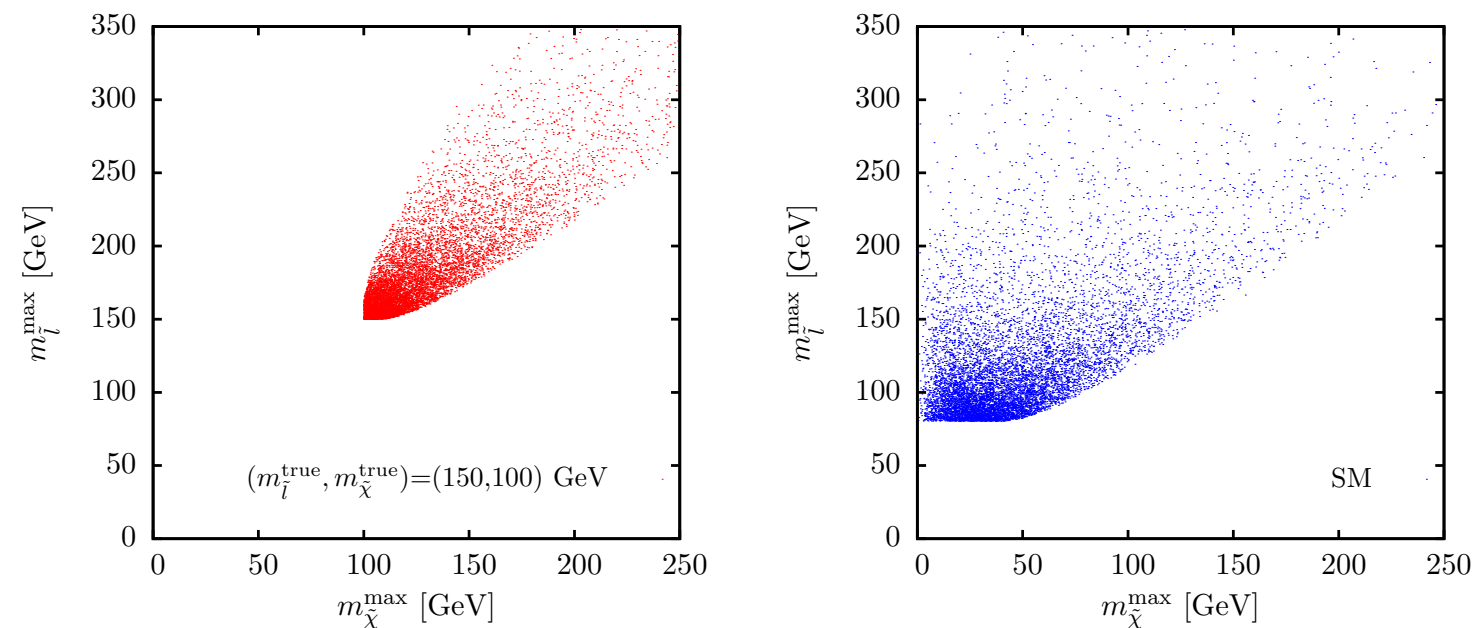

Fig. 3. Scatter plots showing the $\left(m_{\tilde{l}}^{\max }, m_{\tilde{\chi}}^{\max }\right)$ values consistent with each event. Left: SUSY $\left(m_{\tilde{l}}^{\text {true }}, m_{\tilde{\chi}}^{\text {true }}\right)=(150,100)$ GeV. Right: SM. Each plot contains 10,000 events. No detector smearing is included.
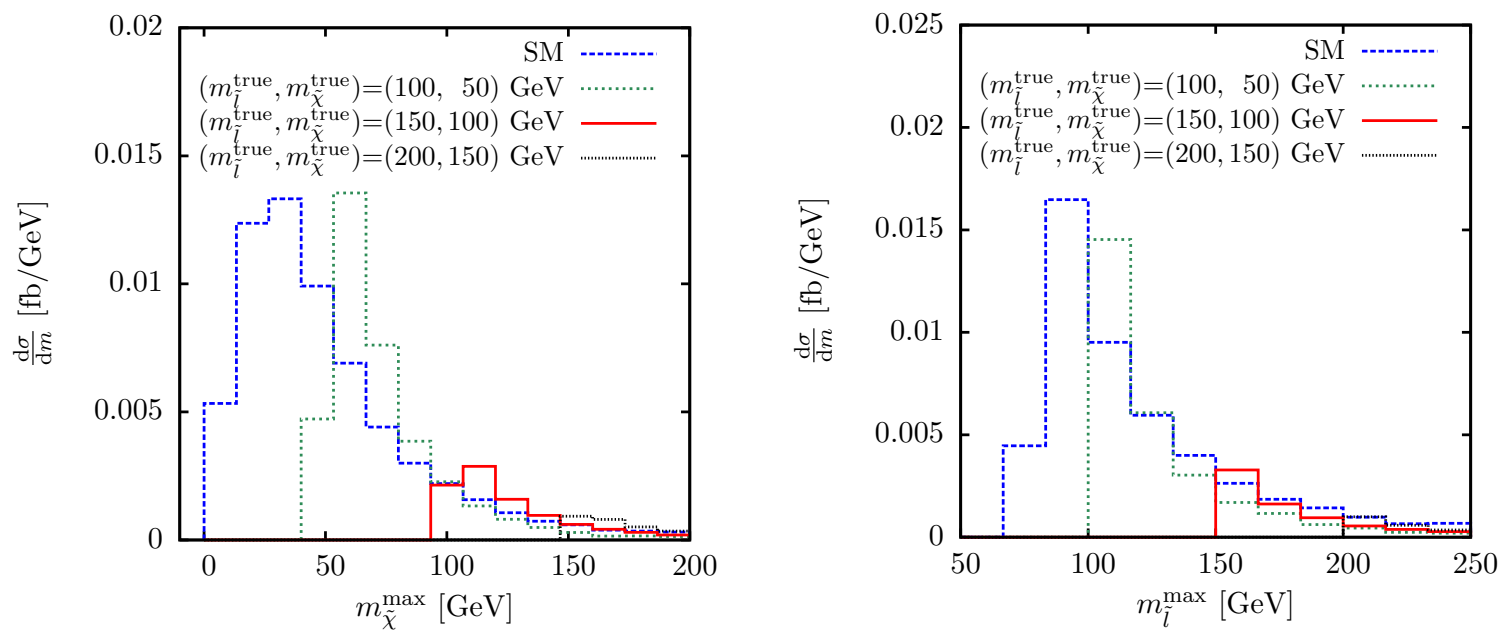

Fig. 4. Histograms showing the $m_{\tilde{\chi}}^{\max }$ (left) and $m_{\tilde{l}}^{\max }$ (right). No detector smearing is included.
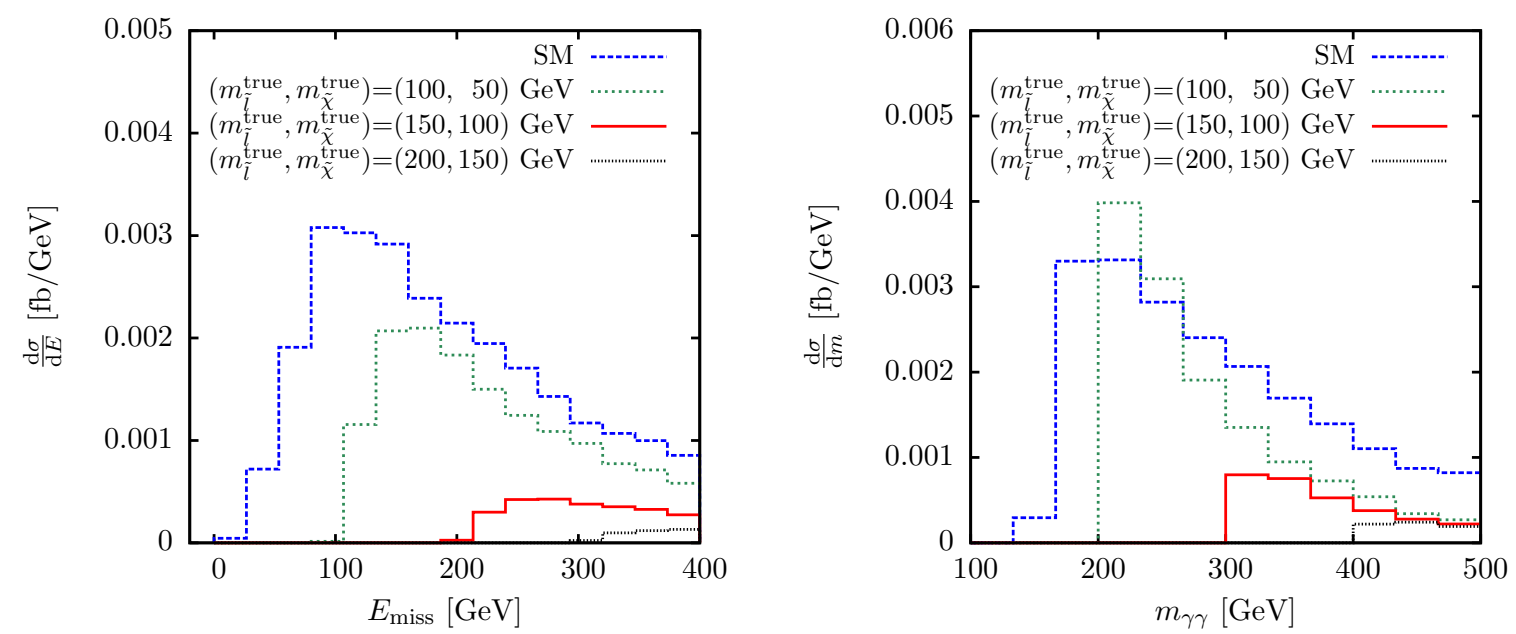

Fig. 5. Histograms showing the $E_{\text {miss }}$ (left) and $m_{\gamma \gamma}$ (right) distributions. No detector smearing included. 
ties in determining the endpoint value. In contrast, the impact on the $m_{\tilde{\chi}}^{\max }$ distribution is relatively mild.

To estimate the precision in determining $\left(m_{\tilde{l}}^{\text {true }}, m_{\tilde{\chi}}^{\text {true }}\right)$ that can be obtained by measuring $\left(m_{\tilde{l}}^{\max }, m_{\tilde{\chi}}^{\max }\right)$ at the LHC, we perform a pseudo-experiment which includes detector smearing effects. The 4 -momenta of the final state leptons are smeared via

$$
\left(\frac{\delta E}{E}\right)^{2}=\frac{a^{2}}{E}+b^{2},
$$

where $a=0.1 \sqrt{\mathrm{GeV}}$ and $b=0.007$ [48, 49]. The fractional momentum loss $\xi$ of the final state protons is smeared vid

$$
\frac{\delta \xi}{\xi}=0.04
$$

In the pseudo-experiment, the number of events we generate for both the SUSY signal, $\left(m_{\tilde{l}}^{\text {true }}, m_{\tilde{\chi}}^{\text {true }}\right)=(150,100$ $\mathrm{GeV}$, and SM background is based on the cross sections displayed in Table [1, assuming an integrated luminosity of $300 \mathrm{fb}^{-1}$, i.e. 216 and 38 events for the SM and SUSY model respectively. We further define the signal region as the range within $m_{\tilde{l}}^{\max }=[130: 230] \mathrm{GeV}$ and $m_{\tilde{\chi}}^{\max }=[80: 180] \mathrm{GeV}$ to increase the $\mathrm{S} / \mathrm{B}$ ratio. In our particular pseudo-experiment sample, 24 and 36 events are observed in the signal region for the SM and SUSY model respectively. We construct the posterior probability density of $\left(m_{\tilde{l}}, m_{\tilde{\chi}}\right)$ if and identify the $68 \%$ and $95 \%$ credibility region as follows. Given a hypothesis $\mathbf{m}=\left(m_{\tilde{l}}, m_{\tilde{\chi}}\right)$, the probability density function (p.d.f.) for the SUSY sample in obtaining $\mathbf{m}^{\max }=\left(m_{\tilde{l}}^{\max }, m_{\tilde{\chi}}^{\max }\right)$, denoted by $\rho_{\mathrm{SUSY}}\left(\mathbf{m}^{\max } ; \mathbf{m}\right)$, is estimated by generating 1 million events, and binning the events passing the cuts into a grid with bin size $2 \mathrm{GeV} \times$ $2 \mathrm{GeV}$ on the $\left(m_{\tilde{l}}^{\max }, m_{\tilde{\chi}}^{\max }\right)$ plane. We generate $1189 \mathrm{SUSY}$ mass points in the range of $m_{\tilde{l}}=[145: 152] \mathrm{GeV}$ and $m_{\tilde{\chi}}=[93: 103] \mathrm{GeV}$ with a $0.25 \mathrm{GeV}$ step size. The p.d.f. for the SM background sample, $\rho_{\mathrm{SM}}\left(\mathbf{m}^{\mathrm{max}}\right)$, is estimated by generating 1 million events in the same way. The combined probability density, $\rho\left(\mathbf{m}^{\max } ; \mathbf{m}\right)$, is then given by

$$
\begin{aligned}
\rho\left(\mathbf{m}^{\max } ; \mathbf{m}\right)= & f_{\mathrm{SM}} \rho_{\mathrm{SM}}\left(\mathbf{m}^{\max }\right) \\
& +f_{\mathrm{SUSY}} \rho_{\mathrm{SUSY}}\left(\mathbf{m}^{\max } ; \mathbf{m}\right),
\end{aligned}
$$

where $f_{\mathrm{SM}}$ and $f_{\mathrm{SUSY}}$ are the fraction of SM and SUSY cross section after the cuts, with $f_{\mathrm{SM}}+f_{\mathrm{SUSY}}=1$.

The likelihood function, i.e. joint p.d.f., may be written as

$$
L(\mathbf{m})=\prod_{i} \rho\left(\mathbf{m}^{\max } ; \mathbf{m}\right)
$$

where $i$ runs over the number of events observed in the signal region. We define the posterior probability density

\footnotetext{
${ }^{4}$ We choose this level of smearing to reproduce the quoted 'missing mass' resolution of roughly $2-3 \mathrm{GeV}$ for a $120 \mathrm{GeV}$ SM Higgs boson 30]
}

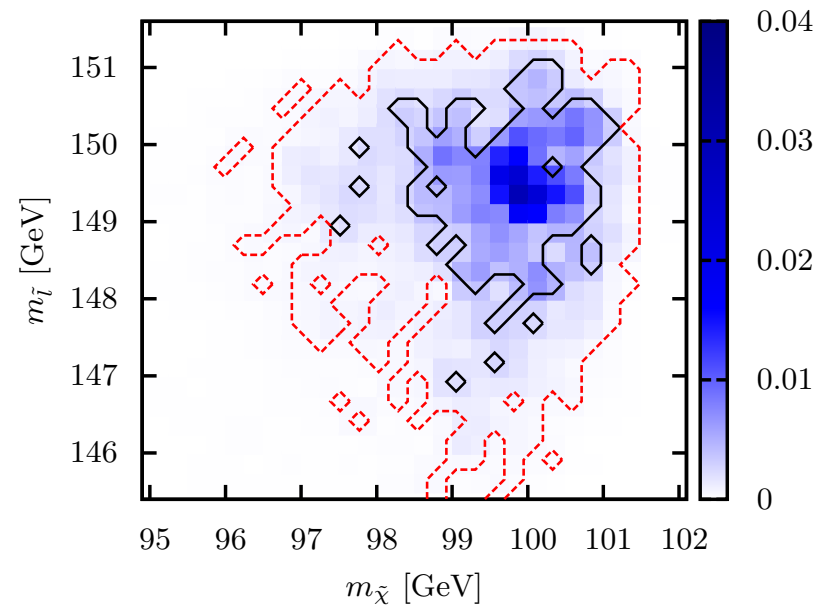

Fig. 6. Credibility distribution for the $\left(m_{\tilde{l}}, m_{\tilde{\chi}}\right)$ hypothesis in a pseudo-experiment. The mass parameters of the SUSY model are given by $\left(m_{\tilde{l}}^{\text {true }}, m_{\tilde{\chi}}^{\text {true }}\right)=(150,100) \mathrm{GeV}$. The solid black łnd dashed red contours contain the $68 \%$ and $95 \%$ credibility regions respectively.

$\mathrm{a} 5$

$$
p(\mathbf{m})=\frac{L(\mathbf{m})}{\int d \mathbf{m}^{\prime} L\left(\mathbf{m}^{\prime}\right)} .
$$

A hypothesis $\mathbf{m}$ is inside the $68 \%$ (95\%) credibility region

$$
\int d \mathbf{m}^{\prime} p\left(\mathbf{m}^{\prime}\right)<0.68(0.95)
$$

where the integration is carried out over $\mathbf{m}^{\prime}$ satisfying $p\left(\mathbf{m}^{\prime}\right) \geq p(\mathbf{m})$.

We show the $p(\mathbf{m}) \Delta m_{\tilde{l}} \Delta m_{\tilde{\chi}}$ distribution $\left(\Delta m_{\tilde{l}}=\Delta m_{\tilde{\chi}}=\right.$ $0.25 \mathrm{GeV}$ ) from an event sample from one pseudo-experiment in Fig. 6. In this particular sample, with 60 events (24 SM background, 36 SUSY signal) passing the selection criteria, $\left(m_{\tilde{l}}^{\text {true }}, m_{\tilde{\chi}}^{\text {true }}\right)$ can be estimated to about $3-4 \mathrm{GeV}$ accuracy at $95 \%$ credibility. Results from other event samples, which assume the same integrated luminosity, show similar level of accuracies. By applying the likelihood calculation on event samples which do not include finite detector resolution effects, we find that the uncertainty is primarily due to statistical fluctuations.

As can be seen in Fig. 6, there is a preference towards the lower mass hypothesis. This is a consequence of the peaking structure of the $\left(m_{\tilde{l}}^{\max }, m_{\tilde{\chi}}^{\max }\right)$ distributions just above $\left(m_{\tilde{l}}^{\text {true }}, m_{\tilde{\chi}}^{\text {true }}\right)$. This implies that a hypothesis $\left(m_{\tilde{l}}, m_{\tilde{\chi}}\right)$ where $m_{\tilde{l}}>m_{\tilde{l}}^{\text {true }}$ and $m_{\tilde{\chi}}>m_{\tilde{\chi}}^{\text {true }}$ is likely to have many signal events in the region where

5 In other words, we take a constant prior p.d.f., $\pi(\mathbf{m})=c$, in the Bayesian posterior p.d.f.

$$
p(\mathbf{m})=\frac{L(\mathbf{m}) \pi(\mathbf{m})}{\int d \mathbf{m}^{\prime} L\left(\mathbf{m}^{\prime}\right) \pi\left(\mathbf{m}^{\prime}\right)} .
$$


$\rho_{\text {SUSY }}\left(\mathbf{m}^{\max } ; \mathbf{m}\right)$ is zero, and therefore they only 'see' the likelihood contribution from $f_{\mathrm{SM}} \rho_{\mathrm{SM}}\left(m_{\tilde{l}}^{\max }, m_{\tilde{\chi}}^{\max }\right)$, which is rather low. On the other hand the likelihood reduces less quickly in the case where $m_{\tilde{l}}<m_{\tilde{l}}^{\text {true }}$ and $m_{\tilde{\chi}}<m_{\tilde{\chi}}^{\text {true }}$ due to the tail of the $m_{\tilde{l}}^{\max }$ and $m_{\tilde{\chi}}^{\max }$ distributions.

\section{Summary and discussion}

In this paper, we have discussed how the additional kinematic information obtained in CEP with tagged forward protons can be used to simultaneously measure the masses of the particles involved in the short decay chain displayed in Eq. (5). We presented a full solution to the system, and found that the distributions of the quantities $m_{\tilde{l}}^{\max }$ and $m_{\tilde{\chi}}^{\max }$, which are the maximum mass values consistent with a given event, are particularly useful in estimating their corresponding true mass values $m_{\tilde{l}}^{\text {true }}$ and $m_{\tilde{\chi}}^{\text {true }}$. We then performed a likelihood analysis to show that for our reference SUSY model, $\left(m_{\tilde{l}}^{\text {true }}, m_{\tilde{\chi}}^{\text {true }}\right)=(150,100) \mathrm{GeV}$, the true masses can be estimated to a few $\mathrm{GeV}$ at $95 \%$ credibility with just $\mathcal{O}(30)$ signal events.

As emphasised in Ref. [37], the possibility to measure central exclusive production in the high luminosity phase of the LHC is crucial to a wide range of processes studied in the literature. However, one potentially important issue which our simulation has not included is the effect of pile-up: at high luminosity there are expected to be multiple overlap events in each bunch crossing which will fill the rapidity gaps between the central system and the outgoing protons. Even so, by making use of the forward proton taggers and the high precision time-of-flight detectors discussed in Ref. [30] to demand that the tagged protons and the opposite-sign lepton pair all point to the same origin, we may hope to suppress the effect of pile-up, given good vertexing resolution. However a more detailed investigation is beyond the scope of the present study.

Nevertheless, we find the level of accuracies obtainable with the small $(\mathcal{O}(30))$ event sample encouraging. The prospects for measuring the masses in the corresponding decay chain in KK scenarios are even better, due to the much higher signal cross section given the same mass parameters, see Fig. 1.

As discussed in the Introduction, the production of a slepton pair, followed by the decay into neutralinos and leptons in an inelastic scattering is clearly possible. As is well-known, the energy and the longitudinal momentum of the hard process cannot be measured in a hadronhadron collision. Compared to the CEP process that we consider, the inelastic process has a much larger cross section. In principle, the MT2 method [4] can be used to determine the true masses by observing a kink structure at the true mass value. This kink structure is formed by events where the two slepton system recoils against initial state radiation and has a non-zero transverse momentum. However the probability that the system has a significant transverse momentum is very low and the kink resides at the smooth tail of the distribution. Once detector smearing effects are included, it is likely that observing such a kink structure will be difficult. In this context, measuring the particle masses in this particular decay chain in CEP with forward proton tagging might be the best, if not the only, way.

Finally, we note that the mass measurement technique presented here could also be used in future linear colliders. A relevant process could be

$$
\begin{aligned}
e^{+} e^{-} \rightarrow & \tilde{l}_{1}^{+} \tilde{l}_{2}^{-}, \\
& \tilde{l}_{1}^{+} \tilde{l}_{2}^{-} \rightarrow l_{1}^{+} l_{2}^{-} \tilde{\chi}_{1} \tilde{\chi}_{2},
\end{aligned}
$$

where the $p_{\gamma \gamma}$ is simply the vector sum of the incoming electron 4-momenta. At parton level, the leptons have a flat energy distribution with endpoints $E_{\max \text { min }}$ at

$$
\begin{aligned}
E_{\max , \min }=\frac{\sqrt{s}}{4} & {\left[1-\left(\frac{m_{\tilde{\chi}}^{\text {true }}}{m_{\tilde{l}}^{\text {true }}}\right)^{2}\right] } \\
\times & {\left[1 \pm \sqrt{1-\left(\frac{2 m_{\tilde{l}}^{\text {true }}}{\sqrt{s}}\right)^{2}}\right], }
\end{aligned}
$$

cf. Ref. [53]. Since the masses are determined by the endpoints, the events away from the endpoints are not directly used. Our $m_{\tilde{l}}^{\max }$ and $m_{\tilde{\chi}}^{\max }$ distributions on the other hand peak strongly near $m_{\tilde{l}}^{\text {true }}$ and $m_{\tilde{\chi}}^{\text {true }}$, so could result in better statistics depending on the relation between $m_{\tilde{\chi}}^{\text {true }}$, $m_{\tilde{l}}^{\text {true }}$ and $\sqrt{s}$. A more detailed comparative study of these two measurement methods can be found in Ref. [44.

Acknowledgements We thank members of the Cambridge SUSY Working Group, particularly Chris Lester, for many useful discussions. This work has been supported in part by the Isaac Newton Trust and the STFC. CHK would like to thank the Particle and Astroparticle Physics group at MPIK Heidelberg and the Rudolf Peierls Centre for Theoretical Physics at Oxford for hospitality while part of the work was carried out. KS is supported in part by the YLC (Young Leaders Cultivation) programme in Nagoya University. LHL acknowledges financial support from the University of Cambridge Domestic Research Studentship scheme. WJS and LHL acknowledge financial support in the form of an IPPP Associateship.

\section{Appendix: differential QED cross sections with $\gamma \gamma$ initial states}

For ease of reference, the differential cross sections for pair production of final state particles for $W^{+} W^{-}$[50], $S^{+} S^{-}[51]$ and $F^{+} F^{-}[52]$ with $\gamma \gamma$ initial states are listed 
below:

$$
\begin{aligned}
\frac{d \sigma\left(W^{+} W^{-}\right)}{d \cos \theta^{*}}= & \frac{\pi \alpha^{2} \beta}{\hat{s}\left(1-\beta^{2} \cos ^{2} \theta^{*}\right)^{2}}\left[19-6 \beta^{2}\left(1-\beta^{2}\right)\right. \\
& \left.+2\left(8-3 \beta^{2}\right) \beta^{2} \cos ^{2} \theta^{*}+3 \beta^{4} \cos ^{4} \theta^{*}\right], \\
\frac{d \sigma\left(S^{+} S^{-}\right)}{d \cos \theta^{*}}= & \frac{\pi \alpha^{2} \beta}{\hat{s}\left(1-\beta^{2} \cos ^{2} \theta^{*}\right)^{2}}\left[\left(1-\beta^{2} \cos ^{2} \theta^{*}\right)^{2}\right. \\
& \left.-2\left(1-\beta^{2}\right)\left(1-\beta^{2} \cos ^{2} \theta^{*}\right)+2\left(1-\beta^{2}\right)^{2}\right], \\
\frac{d \sigma\left(F^{+} F^{-}\right)}{d \cos \theta^{*}}= & \frac{2 \pi \alpha^{2} \beta}{\hat{s}\left(1-\beta^{2} \cos ^{2} \theta^{*}\right)^{2}}\left[-\beta^{4} \cos ^{4} \theta^{*}\right. \\
& \left.+1+2 \beta^{2}\left(1-\beta^{2}\right)\left(1-\cos ^{2} \theta^{*}\right)\right]
\end{aligned}
$$

where $\beta=\left(1-4 m^{2} / \hat{s}\right)^{1 / 2},\left(m=m_{W}, m_{F}, m_{S}\right), \theta^{*}$ is the angle of out-going particles with respect to the $\gamma^{\prime}$ s in the C.M. frame. The charges and colour factors are all assumed to be 1 . The total cross sections are given by

$$
\begin{aligned}
\sigma\left(W^{+} W^{-}\right)=\frac{\pi \alpha^{2} \beta}{\hat{s}}[ & 2 \frac{22-9 \beta^{2}+3 \beta^{4}}{1-\beta^{2}} \\
& \left.-3 \frac{1-\beta^{4}}{\beta} \ln \left(\frac{1+\beta}{1-\beta}\right)\right], \\
\sigma\left(S^{+} S^{-}\right)=\frac{2 \pi \alpha^{2} \beta}{\hat{s}}\left[2-\beta^{2}\right. & \left.-\frac{1-\beta^{4}}{2 \beta} \ln \left(\frac{1+\beta}{1-\beta}\right)\right] \\
\sigma\left(F^{+} F^{-}\right)=\frac{4 \pi \alpha^{2} \beta}{\hat{s}} & {\left[-2+\beta^{2}\right.} \\
& \left.+\frac{3-\beta^{4}}{2 \beta} \ln \left(\frac{1+\beta}{1-\beta}\right)\right] .
\end{aligned}
$$

\section{References}

1. F. E. Paige, [hep-ph/9609373].

2. I. Hinchliffe, F. E. Paige, M. D. Shapiro, J. Soderqvist, W. Yao, Phys. Rev. D55 (1997) 5520-5540. [hep$\mathrm{ph} / 9610544]$.

3. B. C. Allanach, C. G. Lester, M. A. Parker, B. R. Webber, JHEP 0009 (2000) 004. [hep-ph/0007009].

4. C. G. Lester, D. J. Summers, Phys. Lett. B463 (1999) 99-103. [hep-ph/9906349].

5. A. Barr, C. Lester, P. Stephens, J. Phys. G G29 (2003) 2343-2363. [hep-ph/0304226].

6. H. -C. Cheng, Z. Han, JHEP 0812 (2008) 063. [arXiv:0810.5178 [hep-ph]].

7. A. J. Barr, B. Gripaios, C. G. Lester, JHEP 0802 (2008) 014. [arXiv:0711.4008 [hep-ph]].

8. W. S. Cho, K. Choi, Y. G. Kim, C. B. Park, JHEP 0802 (2008) 035. [arXiv:0711.4526 [hep-ph]].

9. M. M. Nojiri, Y. Shimizu, S. Okada, K. Kawagoe, JHEP 0806 (2008) 035. [arXiv:0802.2412 [hep-ph]].

10. M. M. Nojiri, K. Sakurai, Y. Shimizu, M. Takeuchi, JHEP 0810 (2008) 100. [arXiv:0808.1094 [hep-ph]]

11. M. Burns, K. Kong, K. T. Matchev, M. Park, JHEP 0903 (2009) 143. [arXiv:0810.5576 [hep-ph]].
12. M. M. Nojiri, K. Sakurai, Phys. Rev. D82 (2010) 115026. [arXiv:1008.1813 [hep-ph]].

13. K. T. Matchev, F. Moortgat, L. Pape, M. Park, Phys. Rev. D82 (2010) 077701. [arXiv:0909.4300 [hep-ph]].

14. K. T. Matchev, M. Park, Phys. Rev. Lett. 107 (2011) 061801. [arXiv:0910.1584 [hep-ph]].

15. P. Konar, K. Kong, K. T. Matchev, M. Park, Phys. Rev. Lett. 105 (2010) 051802. [arXiv:0910.3679 [hep$\mathrm{ph}]$.

16. A. J. Barr, B. Gripaios, C. G. Lester, JHEP 0911 (2009) 096. [arXiv:0908.3779 [hep-ph]].

17. P. Konar, K. Kong, K. T. Matchev, M. Park, JHEP 1004 (2010) 086. [arXiv:0911.4126 [hep-ph]].

18. G. G. Ross, M. Serna, Phys. Lett. B665 (2008) 212218. [arXiv:0712.0943 [hep-ph]].

19. A. J. Barr, G. G. Ross, M. Serna, Phys. Rev. D78 (2008) 056006. [arXiv:0806.3224 [hep-ph]].

20. A. J. Barr, T. J. Khoo, P. Konar, K. Kong, C. G. Lester, K. T. Matchev, M. Park, [arXiv:1105.2977 [hep-ph]].

21. K. Kawagoe, M. M. Nojiri, G. Polesello, Phys. Rev. D71 (2005) 035008. [hep-ph/0410160].

22. H. -C. Cheng, J. F. Gunion, Z. Han, G. Marandella, B. McElrath, JHEP 0712 (2007) 076. [arXiv:0707.0030 [hep-ph]].

23. H. -C. Cheng, D. Engelhardt, J. F. Gunion, Z. Han, B. McElrath, Phys. Rev. Lett. 100 (2008) 252001. [arXiv:0802.4290 [hep-ph]].

24. B. Webber, JHEP 0909 (2009) 124. [arXiv:0907.5307 [hep-ph]].

25. M. M. Nojiri, K. Sakurai, B. R. Webber, JHEP 1006 (2010) 069. [arXiv:1005.2532 [hep-ph]].

26. H. -C. Cheng, J. Gu, [arXiv:1109.3471 [hep-ph]].

27. A. J. Barr, C. G. Lester, J. Phys. G G37 (2010) 123001. [arXiv:1004.2732 [hep-ph]].

28. M. G. Albrow, T. D. Coughlin, J. R. Forshaw, Prog. Part. Nucl. Phys. 65 (2010) 149-184. [arXiv:1006.1289 [hep-ph]].

29. V. A. Khoze, A. D. Martin, M. G. Ryskin, Eur. Phys. J. C23 (2002) 311-327. [hep-ph/0111078].

30. M. G. Albrow et al. [ FP420 R and D Collaboration ], JINST 4 (2009) T10001. [arXiv:0806.0302 [hep-ex]].

31. F. del Aguila, L. Ametller, Phys. Lett. B261 (1991) 326-333.

32. H. Baer, C. -h. Chen, F. Paige, X. Tata, Phys. Rev. D49 (1994) 3283-3290. [hep-ph/9311248].

33. N. Schul, K. Piotrzkowski, Nucl. Phys. Proc. Suppl. 179-180 (2008) 289-297. [arXiv:0806.1097 [hep-ph]].

34. J. de Favereau de Jeneret, V. Lemaitre, Y. Liu, S. Ovyn, T. Pierzchala, K. Piotrzkowski, X. Rouby, N. Schul et al., [arXiv:0908.2020 [hep-ph]].

35. V. A. Khoze, A. D. Martin, M. G. Ryskin, Phys. Lett. B401 (1997) 330-336. [hep-ph/9701419].

36. V. A. Khoze, A. D. Martin, M. G. Ryskin, Eur. Phys. J. C14 (2000) 525-534. [arXiv:hep-ph/0002072 [hep$\mathrm{ph}]$.

37. P. J. Bussey, T. D. Coughlin, J. R. Forshaw, A. D. Pilkington, JHEP 0611 (2006) 027. [hepph/0607264]. 
38. M. Boonekamp, J. Cammin, S. Lavignac, R. B. Peschanski and C. Royon, Phys. Rev. D 73 (2006) 115011. [arXiv:hep-ph/0506275].

39. S. Heinemeyer, V. A. Khoze, M. G. Ryskin, W. J. Stirling, M. Tasevsky, G. Weiglein, Eur. Phys. J. C53 (2008) 231-256. [arXiv:0708.3052 [hep-ph]].

40. J. R. Forshaw, J. F. Gunion, L. Hodgkinson, A. Papaefstathiou and A. D. Pilkington, JHEP 0804 (2008) 090. [arXiv:0712.3510 [hep-ph]].

41. S. Heinemeyer, V. A. Khoze, M. G. Ryskin, M. Tasevsky and G. Weiglein, arXiv:1106.3450 [hep-ph].

42. K. Belotsky, V. A. Khoze, A. D. Martin and M. G. Ryskin, Eur. Phys. J. C 36 (2004) 503. [arXiv:hep-ph/0406037].

43. The PhoCEP code and documentation are available at http://projects.hepforge.org/superchic/phocep.html.

44. L. A. Harland-Lang, C. H. Kom, K. Sakurai and W. J. Stirling, arXiv:1202.0047 [hep-ph].

45. V. M. Budnev, I. F. Ginzburg, G. V. Meledin, V. G. Serbo, Phys. Rept. 15 (1975) 181-281.

46. K. Nakamura et al. [ Particle Data Group Collaboration ], J. Phys. G G37 (2010) 075021.

47. E. Chapon, C. Royon, O. Kepka, Phys. Rev. D81 (2010) 074003. [arXiv:0912.5161 [hep-ph]].

48. G. L. Bayatian et al. [ CMS Collaboration ], J. Phys. G G34 (2007) 995-1579.

49. E. Richter-Was [ Atlas Collaboration ], Acta Phys. Polon. B40 (2009) 1909-1930. [arXiv:0903.4198 [hep$\mathrm{ex}]$.

50. G. Tupper, M. A. Samuel, Phys. Rev. D23 (1981) 1933.

51. J. Ohnemus, T. F. Walsh, P. M. Zerwas, Phys. Lett. B328 (1994) 369-373. [hep-ph/9402302].

52. S. J. Brodsky, T. Kinoshita, H. Terazawa, Phys. Rev. Lett. 25 (1970) 972-975. S. J. Brodsky, T. Kinoshita, H. Terazawa, Phys. Rev. D4 (1971) 1532-1557.

53. S. P. Martin, In *Kane, G.L. (ed.): Perspectives on supersymmetry* 1-98. [arXiv:hep-ph/9709356 [hep-ph]]. 